\title{
Actores y actantes en los conflictos socioambientales. Las redes del caso Bagua
}

Actors and actants in socio-environmental conflicts. The networks of the Bagua case

Atores e atuantes em conflitos socioambientais. As redes do caso Bagua

María MENDOZA MICHILOT

Universidad de Lima - Perú

tmendoza@ulima.edu.pe

Chasqui. Revista Latinoamericana de Comunicación

$N .^{o}$ 147, agosto-noviembre 2021 (Sección Monográfico, pp. 221-240)

ISSN 1390-1079 / e-ISSN 1390-924X

Ecuador: CIESPAL

Recibido: 25-03-2021 / Aprobado: 10-07-2021 


\title{
Resumen
}

Este artículo se focaliza en las relaciones que las audiencias digitales entablan con los protagonistas de los conflictos socioambientales en el Perú. La muestra son los comentarios espontáneos en YouTube sobre los reportajes referidos a Bagua, conflicto amazónico que desde su estallido en 2009 sigue originando un clima de confrontación. Tomando como base la teoría del actor-red, el análisis de redes y la minería de datos, el estudio se pregunta lo siguiente: ¿qué tipo de vínculos emergen del debate virtual?, ¿cómo se retrata y valoriza la agencia de los actores (humanos) y actantes (no humanos)? Se concluye que los vínculos más importantes se relacionan con las víctimas y victimarios del conflicto, valorizados positiva o negativamente por el rol cumplido en el proceso.

Palabras clave: conflictos socioambientales, YouTube, análisis de redes, minería de datos, actor-red.

\begin{abstract}
This article focuses on relations that digital audiences establish with the protagonists of socio-environmental conflicts in Peru. The samples are spontaneous comments in YouTube regarding the interviews about Bagua, the conflict that started in the Bagua province of the Amazon state in 2009, which keeps causing a climate of confrontation. Taking as its starting point the actornetwork theory, network analysis, and data mining, the research study raises the following questions: What type of relations emerge from virtual debates? How is the agency of actors (humans) and actants (nonhumans) portrayed and valued? It is concluded that the most important relations are in connection with the victims and victimizers of the conflict, who are positively or negatively valued owing to their role in the process.
\end{abstract}

Keywords: socio-environmental conflicts, YouTube, network analysis, data mining, actor-network.

\section{Resumo}

O objetivo deste artigo é discorrer sobre as relações que o público digital estabelece com os protagonistas dos conflitos socioambientais no Peru. As amostras são comentários espontâneos no YouTube a respeito das entrevistas sobre Bagua, o conflito que começou na província de Bagua, estado do Amazonas, em 2009, e que continua causando um clima de confronto. Tomando como ponto de partida a teoria ator-rede, a análise de redes e a mineração de dados, a pesquisa levanta as seguintes questões: que tipo de relações emergem dos debates virtuais? Como a agência de atores (humanos) e actantes (não humanos) é retratada e valorizada? Conclui-se que as relações mais importantes são as relacionadas com as vítimas e vitimizadores do conflito, que são valorizados positiva ou negativamente pelo seu papel no processo.

Palavras-chave: conflitos socioambientais, YouTube, análise de redes, mineração de dados, ator-rede. 


\section{Introducción}

El poder de las redes sociales digitales radica en la interacción, así como en su composición dentro de la esfera donde se debaten los temas de interés público (Rheingold, 2004). Los Estados y las empresas, los activistas transnacionales, los actores no estatales (Castells, 2008) y la comunidad virtual en su conjunto pueden ejercer una influencia significativa en los procesos políticos y no políticos que se libran en la opinión pública.

Camino a la tercera década del siglo XXI, los social media son espejos del mundo offline, en la medida que contribuyen a la representación del mundo real, sus protagonistas y objetos de interés social, que pasan a ser escrutados abiertamente y con mayor celeridad en la esfera pública online (Domínguez, López \& Ortega, 2016). Pero además son complejos entramados de entidades vinculadas, yuxtapuestas, asociadas e interrelacionadas, cada una con una posición determinada (Day, 2019; Callon, Rip \& Law, 1986), que se retroalimenta a partir de transformar, compartir o manipular la información de manera colectiva (González-Palacio, González-Pala \& Ortíz-Pabón, 2017). Pueden ser grupos, clases, campos, individuos racionales o entidades no racionales (Latour, 2008; Prestel, 2006) que pueden vincularse en sus innovaciones, conexiones y experiencias colectivas, siendo importante hacer un seguimiento de su desempeño para reconocer las asociaciones que logran realizar y sobre todo para entender de otra manera lo social (Correa, 2012).

De allí que esta investigación analiza el tipo de relaciones que las audiencias digitales entablaron con las partes involucradas durante el conflicto socioambiental de Bagua. Ubicada en la región Amazonas, al noroeste del Perú, esta provincia fue escenario de un cruento evento de amplia repercusión dentro y fuera del país en junio del año 2009, y que genera controversia hasta la actualidad. Dichos lazos siguen activados o, como diría Latour (Correa, 2012), se reconstruyen poniendo en tela de juicio hechos evidentes e indiscutibles (Li, 2015) sobre la agenda, el movimiento y los actores indígenas amazónicos (Cavero, 2016).

El problema de Bagua, que enfrentó a las comunidades indígenas amazónicas y al gobierno central, puede leerse por etapas o ciclos desde el periodo 20082009, años de escalamiento del conflicto, hasta la actualidad. Ha sido el más largo y el más cruento en lo que va de este siglo. En la confrontación entre manifestantes indígenas y las fuerzas del orden, producida el 5 de junio de 2009 , murieron en el fuego abierto veintitrés policías, cinco civiles indígenas y cinco civiles no indígenas; además, hubo doscientos heridos y un policía hasta hoy desaparecido (Cavero, 2016). El conflicto conmocionó al país y a la comunidad internacional, y puso en evidencia por primera vez la demanda democrática de una comunidad indígena amazónica de ser escuchada frente a disposiciones gubernamentales que afectaban sus territorios, espacios y vida (Rodríguez, 2009). Si bien las normas que originaron el conflicto —orientadas a promover 
la inversión privada en tierras indígenas- fueron desestimadas, subsiste en la memoria colectiva un problema de fondo sin resolverse: la ausencia del Estado para minorías étnicas históricamente olvidadas (Espinoza, 2017). Quienes fueron entonces actores principales son evocados recurrentemente en el debate público, asociados al rol positivo o negativo que cumplieron en el conflicto; asimismo, algunos actantes siguen presentes como testigos inanimados de la deuda social pendiente. Cabe señalar que si bien son múltiples los conflictos producidos en el país (hasta enero de 2021, había 196 conflictos sociales -145 activos y 51 latentes-, de los cuales 128 son socioambientales) (Defensoría del Pueblo, 2021), la mayoría ha pasado desapercibida a excepción del de Bagua.

Esto ha originado que se realicen varias investigaciones sobre el caso y sus impactos (Llique \& Santome, 2019; Zambrano, 2017; Guzmán, 2015, 2017; Guzmán \& Rodrigo Alsina, 2019; Manacés \& Gómez, 2013; Hinojosa, Ricco \& Toasa, 2013; Dolorier \& Paneque, 2013; Guevara, 2011; Surrallés, 2011; Romio, 2010). En este artículo se analiza un aspecto poco abordado: la perspectiva de los ciudadanos y el tipo de relaciones que entablaron con los actores del conflicto a partir de las noticias difundidas en YouTube. A través de esta red social digital, una de las de mayor consumo informativo sobre todo entre las nuevas generaciones (Marinas, 2019), los cibernautas revelaron interés y compromiso con el tema (Antony \& Thomas, 2010). Las preguntas de investigación son las siguientes: ¿cómo retrataron y valorizaron los usuarios la agencia de actores y actantes, de lo humano y lo no humano, involucrados en Bagua?, ¿cuáles son las características de la red de vínculos locales y regionales que se construyeron en la interacción digital?

El trabajo discute sobre los conflictos sociales en la era digital, el concepto de actor-red y las narrativas de su formulación, así como las comunidades virtuales como espejos sociotécnicos, sus mediaciones, performatividades y vínculos, que analiza desde el Análisis de Redes (ARS) y la minería de datos (Social Media Mining, o SMM por sus siglas en inglés).

\section{Conflictividad social en la era digital}

Los conflictos sociales han sido definidos desde dos perspectivas opuestas: de un lado, como resultante del deficiente funcionamiento de las estructuras sociales; de otro lado, como una valiosa oportunidad para forzar la innovación y la creatividad (Coser, 1961). Ambas acepciones clásicas coinciden en que son hechos sociales, tal como los definía Durkheim, es decir, fenómenos centrales en la vida cotidiana que se dan sobre la base de las interacciones de los individuos.

Los problemas se construyen histórica, cultural y sobre todo socialmente (Latour, 2008), y en el caso de los socioambientales, las partes involucradas navegan en estrategias de negociación y coerción, entre el consenso y el disenso, lo cual puede afectar a las siempre cambiantes relaciones entre poblacionesgrupos y quienes ostentan el poder, así como a todo el sistema social (Collins, 
1996; Pérez Castro, 2005; Caballero, 2009). No obstante, la lucha por la tierra, el agua y los bosques (Buckles, 2000) ofrece la oportunidad no solo de disputar, sino de producir y transmitir algún tipo de conocimiento que dependerá de quién es el actor y de su experiencia. Se entiende por conocimiento aquella producción que trasciende a la visión personal de alguien que es producto de la conexión de personas, redes, organizaciones, instituciones y estrategias que garantizan propuestas y cambios viables (Parra-Romero, 2020).

En la sociedad del riesgo en que vivimos (Beck, 1998), los conflictos socioambientales suelen percibirse como algo que afecta a la naturaleza y a la sociedad (Leff, 2011; Lezama, 2004), aunque subsisten algunas lecturas individualistas. Así, por ejemplo, lo apolítico puede tornarse político, y surgir la categoría de "los otros", pese a que en una sociedad del riesgo todos somos afectados directa o indirectamente. El impacto de la contaminación de recursos y territorios - graves, incalculables e irreversibles - puede prevenirse, aunque puede haber tensiones entre la supresión del riesgo y el negocio, entre los afectados y los que se benefician con el problema (Beck, 1998).

Grandes movimientos se han forjado durante la última década en la virtualidad de los medios sociales. Convertidos en espacios autónomos, independientes de todo control político o empresarial (Castells, 2014), estos han permitido exponer aristas que pasaron desapercibidas en otros canales de comunicación pese a su relevancia y necesidad de consensos (Carty, 2011; Carty, 2015). Así, algunas redes digitales han demostrado que pueden ir más allá y convertirse en el escenario propicio para que los actores involucrados en los conflictos no solo se expresen, sino para que su participación se traduzca en la producción y movilización de algún tipo de conocimiento, sea científico, activista o local/comunitario (Parra-Romero, 2020).

En el plano de la participación de actores más vinculados al ámbito local y comunitario, Adela Parra-Romero (2020) plantea que podrían transmitir los siguientes tipos de conocimiento: contrainformativo (la historia no contada), de interpretacióncrítica(evalúaycuestionalainformaciónoficial),visionarioradical y transformativo (plantea alternativas de cambio), de desafío del conocimiento hegemónico (disrupción respecto a lo aceptado académicamente), movilizador de un conocimiento alternativo (plantea estrategias de cambio institucional, político y de comunicación), de empoderamiento de organizaciones de base (capacidades comunitarias y de activismo), de construcción de solidaridades (a través del diálogo con otros autores), de creación de espacios de reflexión (para promover nuevas ideas), entre otros.

\subsection{La vinculación de lo humano y lo no humano}

El papel de las redes sociales como productoras y movilizadoras de conocimiento se enmarca dentro de la ecología política, disciplina que, a través de la economía política y una ejemplar preocupación ambiental, ha logrado poner sobre la 
mesa las relaciones desiguales de poder que dan origen a movimientos sociales, las agencias de los actores involucrados y los conflictos ontológicos ( $\mathrm{Li}, 2015$; Martínez-Alier, 2015). Marcos analíticos influyentes, como la maldición de los recursos naturales, la justicia ambiental y el giro o la apertura ontológica, identifican los conflictos y movimientos sociales como productores de conocimiento contrahegemónico, movilizadores de corrientes y discursos que cuestionan modelos, plantean prácticas alternativas de desarrollo y de organización (Tarabini, 2019).

Para este artículo, es pertinente referirse a la teoría del actor-red (TAR), uno de los enfoques del giro ontológico, que incide en la estrecha relación naturaleza y sociedad o socio-naturaleza: una nueva entidad que valora la heterogeneidad, el entramado de lo técnico y lo social, así como la interacción, relación y asociación de colectivos formados por actores humanos y no humanos en una red, más allá de vínculos tradicionales (Correa, 2012). La TAR considera que el sentido de pertenencia está en crisis, que las realidades sociales no son fijas ni limitadas, sino algo en movimiento, en constante "reasociación y reemsamblado" (Latour, 2008, p. 21).

Desde este enfoque, el ambiente y sus elementos constitutivos no son meros recursos estáticos, sino relaciones entre un colectivo de actores $(\mathrm{Li}, 2015)$ y actantes (Fontanille, 2001). Los primeros son seres humanos que pueblan el mundo social, los segundos son entidades no humanas capaces de generar una relación, adquirir una significación o producir una acción (Correa, 2012). Ambos tienen un programa de acción o capacidad de "hacer", coexisten en las relaciones colectivas, cumplen una función o representación dentro de una escena narrativa, tienen agencia e inciden en la acción de otro actor o actante. $\mathrm{Su}$ presencia explica las diferencias, las asimetrías, el ejercicio del poder (Latour, 2008). Crean estabilidad o inestabilidad, emergen de prácticas y formas de actuar en algunos casos históricas, producto de acuerdos sociales, técnicos y con el poder (Li, 2015). En América Latina pueden marcar la existencia de dos mundos (De la Cadena, 2015).

\subsection{Redes como espejos sociotécnicos}

Más allá de la necesidad que tienen de exteriorizar las conciencias personales, las redes electrónicas se asemejan a un sistema nervioso que se acopla con sus interconexiones a millones de individualidades (De Kerckhove citado por Baldessar \& Giglio, 2011). La acción colectiva de estas redes es contenciosa (Tarrow, 1997), se expresa en el campo de la subpolítica (Sánchez-Duarte \& Fernández-Romero, 2017) y suele actuar desde la resistencia, aunque en ocasiones sin demandas maduras (Mendoza \& Valenzuela, 2020) o con dificultades para vincular lo online con lo offline.

Plataformas como YouTube, Twitter o Facebook pueden ser espejos sociotécnicos, si vinculan tecnología y entramado social; si establecen 
asociaciones de actantes y actores, y cumplen el papel de canales tecnológicos en las acciones y prácticas cotidianas de las personas (Correa, 2012). Es importante destacar su valor relacional, es decir, de qué manera los gestores y usuarios se vinculan para alcanzar determinados objetivos consensuados o no consensuados (De Kerckhove, s/f; Alonso, 2005).

Como explica Martins (2009), hoy existen dos lecturas sobre las redes sociales: una, simplificadora del fenómeno, que destaca la idea de red como una metáfora y la articulación entre diversas unidades que intercambian elementos, se fortalecen y pueden multiplicarse (Mance citado por Martins, 2009). La otra lectura propone teorizar y sistematizar el concepto de red a partir de las funciones y agencias de sus miembros para dos finalidades: entender las redes como sociotécnicas, es decir, como lazos que vinculan cultura y sociedad, o lo humano con lo no humano (Latour, 2008).

En este estudio, se aplica la segunda lectura. Además, el ARS (o Network Analysis), que recupera el concepto sociológico de estructura social de Simmel (1986), sirve para mostrar la conexión que se puede dar en los social media, entre corporaciones, Estados y sistemas mundiales, entre egos y alteri (Wellman, 1997; Gascue, 2009). Los marcos teóricos, así como los métodos y medidas del ARS - centralidad, conectividad, densidad, entre una variedad de opciones(Christakis \& Fowler, 2009; Granovetter, 1973; Cachia, 2010), permiten revalorar las relaciones o vínculos entre los nodos de una red (González, 2014).

\section{Metodología}

\subsection{Diseño del estudio}

El objeto de estudio son los actores y actantes (egos) involucrados en el conflicto de Bagua, su identificación por parte de las audiencias digitales (alteri), así como el valor relacional de las redes y las vinculaciones que entablan. Se utiliza un método analítico, porque a partir de los datos proporcionados en los comentarios - motivados a su vez por la información periodística propaladase establecen las valoraciones y las relaciones entre egos y alteri.

Entre las técnicas aplicadas, se hace un análisis de la interacción para evaluar de manera cuantitativa la métrica de la plataforma con base en la SMM. Desde lo cualitativo, se observa la temática de los comentarios, tipos y tonos. Se replica el modelo de Orbe y De Kenefuchi (citado por Antony \& Thomas, 2010), que identifica las reacciones del público frente a contenidos audiovisuales, para reconocer en los comentarios el nivel de compromiso del usuario con el debate público (las acciones consecuentes que emprende y con qué finalidades: debatir, organizar, movilizar).

Luego, el ARS permite medir la categoría centralidad (Del Fresno, 2014) y sus indicadores: degree (grado, con valores de o a 1), que indica el número de conexiones de un nodo y aproximarse a su influencia en la red; betweenness 
(grado de intermediación), que cuenta las veces que un nodo aparece en el camino más corto que conecta a otros nodos; y eigenvector, que identifica los nodos que son el centro de grupos cohesivos o que están conectados a nodos a su vez bien conectados.

\subsection{Universo y muestra}

La evidencia empírica es la totalidad de comentarios voluntarios y espontáneos, difundidos entre el 5 de junio de 2009 y el 21 de febrero de 2021, sobre tres reportajes amplificados en YouTube. Los reportajes sobre Bagua fueron propalados inicialmente en programas televisivos de medios influyentes de alcance nacional, así considerados en los buscadores de Alexa, TopSitesWebs y Comscore, como Canal N, Latina (programa Punto final) y América Televisión (programa Cuarto poder). La selección se hizo previa inmersión y monitoreo en YouTube, en enero del año 2020, para definir una muestra significativa bajo dos criterios básicos: a) que estuviera disponible; b) que contara con comentarios recientes. Al respecto, cabe señalar que los videos originales no eran visibles al momento del estudio, por lo que solo se pudo capturar tres copias de reportajes, que reunían los requisitos establecidos y que habían sido propalados por los medios seleccionados en otros canales digitales (Tabla 1).

El análisis de la muestra se realizó en tres etapas:

1. Para el análisis de la interacción se utilizó el software http:// ytcomments.klostermann.ca/ con el fin de recoger la métrica (número de visualizaciones, de me gusta y no me gusta) y extraer los comentarios sobre los videos considerados en el estudio. A partir del universo de comentarios disponibles $(\mathrm{N}=339)$, se determinó una muestra $(\mathrm{N}=209)$ con un margen de error del $5 \%$ y nivel de confianza del 95\% (Tabla 2).

2. Para el análisis de los comentarios, estos se colocaron en la plataforma cualitativa NVivo para ponderar la tematización más frecuente. Luego, se categorizaron en opinativos o informativos, inquisitivos o complacientes, así como insultantes (trol), fuera de contexto (otro tema), propositivos, de convocatoria y discriminadores. Asimismo, se identificó el tono de los comentarios para recoger posiciones a favor (AF), en contra (EC) o neutrales $(\mathrm{N})$ respecto de los actores involucrados y de la noticia o cobertura dada por los medios de comunicación.

3. Para el análisis de redes, se exportó la muestra de comentarios como una base nueva a un fichero del software Ucinet, plataforma de Lin Freeman, Martín Everett y Steve Borgatti, que facilita la construcción de matrices ponderadas de las redes y la visualización de estas en grafos. Ello hizo posible la medición de la categoría centralidad y sus indicadores. 
Tabla 1

\begin{tabular}{|l|l|l|l|l|}
\hline \multicolumn{1}{|c|}{ Videos en YouTube (amplificación de programas TV) } & \multicolumn{1}{|c|}{$\begin{array}{c}\text { Medio/ } \\
\text { Código }\end{array}$} & \multicolumn{1}{|c|}{ Emisión } & \multicolumn{1}{|c|}{$\begin{array}{l}\text { Último } \\
\text { mensaje }\end{array}$} & \multicolumn{1}{|c|}{ Visualizac. } \\
\hline https://www.youtube.com/watch?v=w05nZd1Y9Co & Canal N/cN & 5.6 .09 & 21.2 .21 & 62.757 \\
\hline https://www.youtube.com/watch?v=gXw8LMWH9-A & $\begin{array}{l}\text { Latina-Punto } \\
\text { final/c2 }\end{array}$ & 7.6 .09 & 24.3 .19 & 25.848 \\
\hline https://www.youtube.com/watch?v=sgmdZLbEzE0 & $\begin{array}{l}\text { A m é r i c a - } \\
\text { Cuarto poder/ } \\
\text { c4 }\end{array}$ & 14.6 .09 & 24.3 .13 & 868 \\
\hline
\end{tabular}

Fuente: Elaboración propia

Tabla 2

\begin{tabular}{|l|l|l|l|}
\hline & Universo & Muestra & Unidad de análisis \\
\hline Red Bagua & 339 comentarios & 209 comentarios & Red total \\
\hline
\end{tabular}

Fuente: Elaboración propia

\section{Resultados}

\subsection{Principales actores y actantes}

En el presente análisis, se observa que, de acuerdo con su frecuencia, los actores fueron más referenciados que los actantes. Sin embargo, mientras los actores más importantes fueron nueve de un total de veintitrés entidades, los actantes llegaron a catorce. Se identificaron cuatro grupos o categorías de actores y actantes con el siguiente programa de acción:

1. Actores principales, víctimas y victimarios directos. En este grupo se incluyen a los indígenas de las comunidades amazónicas de Bagua, al expresidente que estuvo en el poder durante el estallido de la crisis (Alan García) y a los policías muertos en el enfrentamiento; también, a todas las víctimas del conficto (indígenas y policías) y a sus victimarios o "asesinos".

2. Actantes principales, escenarios y responsables indirectos. Aparecen estas entidades: el Perú, el pueblo y la selva, como escenarios y testigos del conflicto; y el gobierno y el poder, como responsables del estallido de la crisis.

3. Otros actores políticos: se involucra a líderes amazónicos (Alberto Pizango), líderes de la oposición política (Ollanta Humala) y personajes o grupos politizados que se aduce que estuvieron detrás de las protestas.

4. Otros actantes: menos frecuentes fueron las referencias a los recursos naturales, valores en juego, grupos ideologizados y estereotipos (Tabla 3). 
Tabla 3

\begin{tabular}{|l|l|l|l|l|l|}
\hline Referencias & Porcentaje & Actor/ & Referencias & Porcentaje & Actor/ \\
\hline & ponderado (\%) & Actante & & ponderado (\%) & Actante \\
\hline Indígenas & 1,81 & Actor & Masacre & 0,29 & Actante \\
\hline Perú & 1,19 & Actante & Lima & 0,29 & Actante \\
\hline Alan García P. & 1,11 & Actor & Verdad & 0,27 & Actante \\
\hline Pueblo & 1,01 & Actante & Protesta & 0,26 & Actante \\
\hline Policías & 0,90 & Actor & Diálogo & 0,25 & Actante \\
\hline Gobierno & 0,86 & Actante & Pobreza & 0,24 & Actante \\
\hline Muertos & 0,71 & Actor & Violencia & 0,24 & Actante \\
\hline Asesino & 0,56 & Actor & Desarrollo & 0,24 & Actante \\
\hline Selva & 0,46 & Actante & Matar & 0,23 & Actante \\
\hline Poder & 0,41 & Actante & Aprista & 0,23 & Actante \\
\hline Comunistas & 0,41 & Actor & Indios & 0,23 & Actor \\
\hline Alberto Pizango & 0,40 & Actor & Ignorancia & 0,22 & Actante \\
\hline Progreso & 0,40 & Actante & Ollanta Humala & 0,20 & Actor \\
\hline Partidos & 0,39 & Actante & Socialistas & 0,20 & Actor \\
\hline Tierras & 0,35 & Actante & Terroristas & 0,18 & Actor \\
\hline Ley & 0,30 & Actante & Salvajes & 0,18 & Actor \\
\hline Hermanos & 0,29 & Actante & Hugo Chávez & 0,14 & Actor \\
\hline
\end{tabular}

Fuente: Elaboración propia

\subsection{Retratos}

Según la narrativa de la red, el análisis cualitativo de los comentarios arrojó información complementaria respecto a la forma en que los actores y actantes fueron retratados.

En primer lugar, se observa un mayor número de comentarios inquisidores y cuestionadores, basados en expresiones adjetivas y no necesariamente fundamentadas. Sin embargo, casi en una misma proporción, se hallan comentarios opinativos basados en una argumentación mayor tanto sobre los actantes como sobre los actores.

Luego, aunque en menor número, aparecen los comentarios discriminadores, que recurren a algún tipo de diferenciación de las personas (sexo, color de la piel, nivel socioeconómico, nivel educativo); después, los insultantes (trol) y los que estuvieron fuera del contexto de la conversación (FC). Finalmente, siguen los comentarios convocantes o que sugerían una toma de posición frente al conflicto, y los propositivos, con planteamiento de una solución al problema, 
pero que fueron casi inexistentes. No hubo comentarios complacientes, que mostraran predilección por alguna de las partes sin justificación.

Respecto al tono de los comentarios, se observa de manera predominante los calificativos negativos sobre la gestión de todas las partes involucradas, pero, en especial, de los actores principales (los indígenas y el gobierno de Alan García Pérez). Es más, las referencias negativas hacia el mundo amazónico prevalecen sobre las otras. Además, las referencias negativas hacia la prensa y la cobertura de la noticia fueron mínimas (Tabla 4).

De acuerdo con la clasificación de Orbe y Kenefuchi (citados por Antony \& Thomas, 2010), los internautas buscaron debatir sobre la información difundida, pero sin asumir compromisos mayores respecto al planteamiento de propuestas. Bajo fue el porcentaje de comentarios convocantes a participar en un diálogo nacional o en movilizaciones de protesta por lo sucedido en Bagua. De alguna manera, estos resultados explicarían que los mensajes discriminatorios fueran el tercer tipo de comentarios más frecuente entre los participantes.

Tabla 4

\begin{tabular}{|l|l|l|l|l|l|}
\hline \multicolumn{2}{|c|}{ Tipo de comentarios } & \multicolumn{5}{c|}{ TONOS } & \\
\hline & & La protesta & & La prensa & \\
\hline FC & $10,0 \%$ & EC de Policía & $6,0 \%$ & AF & $1,4 \%$ \\
\hline Trol & $12,0 \%$ & EC FF.AA. & $0,5 \%$ & EC & $2,0 \%$ \\
\hline Inquisitivo & $29,0 \%$ & EC de inversión & $2,0 \%$ & & \\
\hline Opinativo & $27,0 \%$ & EC indigenas & $27,0 \%$ & & \\
\hline Convocante & $7,0 \%$ & EC izquierda 0,5\% & & & \\
\hline Complaciente & & EC de políticos & $7,0 \%$ & & \\
\hline Propositivo & $0,5 \%$ & EC Gobierno (Alan García) & $22,0 \%$ & & \\
\hline Informativos & & EC de protesta & $2,0 \%$ & & \\
\hline Discriminador & $18,0 \%$ & AF protesta & $4,0 \%$ & & \\
\hline & & AF indígenas & $9,0 \%$ & & \\
\hline & AF inversión & $0,5 \%$ & & \\
\hline & AF Gobierno & $\begin{array}{l}10,0 \% \\
19,0 \%\end{array}$ & & \\
\hline
\end{tabular}

Fuente: Elaboración propia

\subsubsection{Estructuras relacionales}

El ARS permitió medir la centralidad de la red a través de los indicadores degree, betweenness y eigenvector (Tabla 5). Respecto a la centralidad, el estudio señala que el nodo más importante o central (degree) fue indígenas, que representa las 
menciones a los actores principales de la crisis. Este acumuló la mayor cantidad de vínculos $(12,2 \%)$.

Además de este nodo, otros actores y actantes destacan en la red y se convierten en puntos de conexión con indígenas y otros nodos. Es el caso de Perú $(12,1 \%)$, pueblo $(9,4 \%)$, Alan García $(7,5 \%)$, policías $(7,1 \%)$ y gobierno $(6,8 \%)$ (Tabla 6).

En cuanto al betweenness, o grado de intermediación, que cuenta las veces que un nodo aparece en el camino más corto que conecta a otros nodos, se ubica en primer lugar nuevamente a Perú, actante que es escenario y testigo de los hechos y del debate público en las redes. Finalmente, el indicador eigenvector, que identifica a los nodos que son el centro de grupos cohesivos o que están conectados a nodos a su vez bien conectados, arroja la supremacía de indígenas.

\section{Tabla 5}

\begin{tabular}{|l|c|c|c|}
\hline EGO & Degree & Betweenness & Eigenvector \\
\hline Indígenas & 0.341 & 0.234 & 0.497 \\
\hline Perú & 0.337 & 0.257 & 0.454 \\
\hline Pueblo & 0.264 & 0.175 & 0.354 \\
\hline Alan García & 0.209 & 0.131 & 0.239 \\
\hline Policías & 0.198 & 0.086 & 0.308 \\
\hline Gobierno & 0.19 & 0.098 & 0.271 \\
\hline Muertos & 0.176 & 0.069 & 0.267 \\
\hline Asesinos & 0.154 & 0.063 & 0.216 \\
\hline Pizango & 0.11 & 0.042 & 0.132 \\
\hline Ignorancia & 0.106 & 0.058 & 0.11 \\
\hline Selva & 0.099 & 0.052 & 0.099 \\
\hline Ollanta Humala & 0.088 & 0.023 & 0.105 \\
\hline Progreso & 0.073 & 0.034 & 0.071 \\
\hline Político/Partidos & 0.066 & 0.015 & 0.09 \\
\hline Comunista/Socialista & 0.062 & 0.028 & 0.046 \\
\hline Tierras & 0.059 & 0.02 & 0.063 \\
\hline Aprista & 0.055 & 0.024 & 0.05 \\
\hline Hugo Chávez & 0.051 & 0.028 & 0.045 \\
\hline Salvajes & 0.048 & 0.022 & 0.032 \\
\hline Terroristas & 0.04 & 0.017 & 0.041 \\
\hline Lima & 0.037 & 0.017 & 0.026 \\
\hline Poder & 0.033 & 0.017 & 0.02 \\
\hline
\end{tabular}

Fuente: Elaboración propia 
Para el análisis de la red Bagua, se asignó a cada comentario un usuario diferente, el cual se identificó con un código —un número seguido del nombre del medio que produjo el reportaje: $\mathrm{cN}$ (Canal N), c2 (Latina-Punto final) y c4. (América TV-Cuarto poder)—. Los vínculos se generaron a partir de cada comentario o, más precisamente, desde el actor o actante mencionado.

La visualización de dichos vínculos da cuenta, de manera significativa, de la centralidad de los egos (actores y actantes) y la valorización que de ellos hicieron los alteri.

Como se observa en el grafo de la red Bagua, esta emerge más nutrida en torno a los nodos principales: indígenas y Perú (Figura 1).

\section{Figura 1}

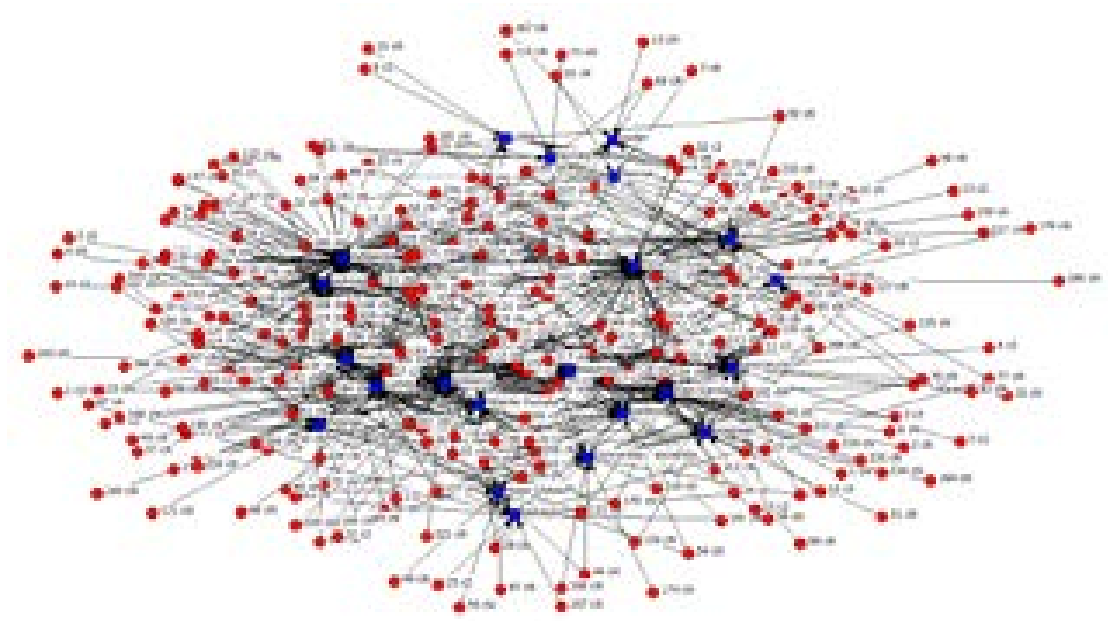

Fuente: Elaboración propia

Tabla 6

\begin{tabular}{|l|l|l|}
\hline Actores y actantes & Lazos (n.) & Lazos (\%) \\
\hline Indígenas & 93 & 12,2 \\
\hline Perú & 92 & 12,1 \\
\hline Pueblo & 72 & 9,4 \\
\hline Alan García & 57 & 7,5 \\
\hline Policías & 54 & 7,1 \\
\hline Gobierno & 52 & 6,8 \\
\hline Muertos & 48 & 6,3 \\
\hline Asesinos & 42 & 5,5 \\
\hline Pizango & 30 & 3,9 \\
\hline Ignorancia & 29 & 3,8 \\
\hline
\end{tabular}




\begin{tabular}{|l|l|l|}
\hline Selva & 27 & 3,5 \\
\hline Ollanta Humala & 24 & 3,1 \\
\hline Progreso & 20 & 2,6 \\
\hline Político & 18 & 2,4 \\
\hline Comunista/Socialista & 17 & 2,2 \\
\hline Tierras & 16 & 2,1 \\
\hline Aprista & 15 & 2,0 \\
\hline Hugo Chávez & 14 & 1,8 \\
\hline Salvajes & 13 & 1,7 \\
\hline Terroristas & 11 & 1,4 \\
\hline Lima & 10 & 1,3 \\
\hline Poder & 9 & 1,2 \\
\hline TOTAL & 763 & 100 \\
\hline
\end{tabular}

Fuente: Elaboración propia

\section{Discusión y conclusiones}

La red del caso Bagua, como se ha denominado en este trabajo al entramado de vínculos de las audiencias digitales (alteri) con los actores y actantes del conflicto (egos), refleja un mundo lleno de relaciones contradictorias.

En principio, debe precisarse que los usuarios son audiencias no especializadas, principalmente citadinas y público objetivo de programas televisivos que, si bien tienen unalcance nacional, están dirigidos principalmente a los habitantes de las ciudades.

El análisis revela que estos alteri se hallan divididos entre aquellos que cuestionan la intervención policial en Bagua y aquellos que vieron en este cruento caso cómo los derechos de las comunidades indígenas se vieron conculcados. Lo interesante es comprobar que, pese al tiempo transcurrido, los vínculos con los nodos importantes (las comunidades amazónicas y el gobierno central) siguen activados o se han reconstruido en el tiempo (Latour, 2008), probablemente porque las causas del conflicto no se han resuelto.

Las redes sociales digitales analizadas se visualizan como espejos sociotécnicos, no solo en la medida que son espacios para disputar asuntos de interés público, sino porque permiten producir algún tipo de intercambio de conocimiento. En el caso que se analiza, se observa que el interés de los alteri se queda en el plano contrainformativo y de interpretación crítica principalmente. No hay un afán disruptivo, alternativo, movilizador o de empoderamiento (Parra-Romero, 2020) de las organizaciones indígenas amazónicas.

Desde la TAR, sin embargo, podría decirse que estas redes digitales ponen la tecnología al servicio de colectivos constituidos por actores y actantes que logran interactuar, relacionarse y asociarse, a pesar de que el sentido de pertenencia 
está en crisis. Frente a realidades sociales que no son fijas ni limitadas, abren un campo hacia la reasociación y el reemsamblado (Latour, 2008).

El análisis realizado indica que los actores y actantes involucrados están presentes en el debate virtual. Sin embargo, en el caso de los actantes (un país, un pueblo, víctimas y victimarios, la selva, el poder, el diálogo, los estereotipos, el progreso, los principios, la pobreza y la violencia) hay una subvaloración. Se les ubica en un segundo plano, su agencia no es reconocida plenamente y tampoco su incidencia en otros actores y actantes. El futuro de las tierras de las comunidades amazónicas y la subsistencia de estas últimas, así como el abandondo ancestral en el que han vivido por décadas, fueron el telón de fondo de la crisis de Bagua, pero no aparecen ni se les destaca en la conversación respecto a la solución del problema.

Por el contrario, la lectura predominante es adjetiva, poco profunda y discriminadora, como lo demuestra el hecho de que las referencias negativas hacia el mundo amazónico son mayores que las positivas o neutrales.

Se confirma, como señala Marisol de la Cadena, que en América Latina algunos actores y actantes no existen para la política, tampoco para el mundo occidental, la herencia colonial ni, habría que añadir, para miles de ciudadanos. Más bien, indica, asistimos al choque de dos mundos, uno de los cuales cuenta con más y el otro, con menos; mundos que si no acatan esta división no son reconocidos como tales. Sus relaciones son de competencia, algunos ganarán la tierra, entendida para muchos como un recurso del Estado y para otros como su vida (2015).

Finalmente, el ARS da cuenta de la heterogeneidad de la comunidad virtual, en la que sin embargo se prioriza a los actores sobre los actantes. En este último caso, cabe resaltar que el Perú aparece como actante, como escenario y también como conglomerado en evolución, cuyo programa de acción se ubica en el campo de la expectativa y la preocupación.

En conclusión, podría decirse que las redes sociales digitales, como la red del caso Bagua, son parte importante en la vida de un país, entre otras razones, porque pueden contribuir a la creación de las identidades (Marinas, 2019), pero también a la formación de un actor-red. Como lo definieron Bruno Latour, Michel Callon y John Law, esta estructura de actores humanos y no humanos, de entidades yuxtapuestas, de materialidades, relaciones, tecnologías y discursos que deben estar ligados y ensamblados (Murray-Li, 2014), amerita ser estudiada y revalorada en sus asociaciones y agencias en temas tan complejos como los conflictos sociales, para reconocer cómo nos visualizamos y cómo incidimos en los otros (Latour, 2008) en situaciones de crisis y polarización social (Murray-Li, 2014). 


\section{Referencias bibliográficas}

Alonso, J. (2005). El sentido de comunidad en los nuevos medios. Razón y palabra(42). Recuperado de http://old.razonypalabra.org.mx/anteriores/n42/jalonso.html

Antony, M. G., \& Thomas, R. (2010). 'This is citizen journalism at its finest': YouTube and the public sphere in the Oscar Grant shooting incident. New Media E Society. doi:10.1177/1461444810362492

Baldessar, M., \& Giglio, K. (2011). Reseña de "The Skin of Culture" de Derrick De Kerckhove. Razón y Palabra, 75. Recuperado de https://www.redalyc.org/articulo. oa?id=199518706007

Beck, U. (1998). La sociedad del riesgo. Barcelona: Paidós.

Buckles, D. (200o). Cultivar la paz. Conflicto y colaboración en el manejo de los recursos naturales. CIID.

Caballero, V. (2009). Los conflicto sociales y socioambientales en el sector rural y su relación con el desarrollo rural. Notas para un balance de investigaciones. Sepia XII.

Cachia, R. (2010). Las redes personales a la luz del análisis de redes sociales. (J. Molina, \& I. Maya Jariego, edits.) Redes, 7-30.

Callon, M., Rip, A., \& Law, J. (1986). Mapping the Dynamics of Science and Technology: Sociology of Science in the Real World. Londres: The MacMillian Press.

Carty, V. (2011). Wired and Mobilizing: Social movements, new technology, and electoral politics. Londres: Routledge.

Carty, V. (2015). Social Movements and New Technology. Nueva York: Westiview Press.

Castells, M. (1 de marzo de 2008). The New Public Sphere: Global Civil Society, Communication Networks, and Global Governance. Sage Journals. doi:https://doi. org/10.1177/0002716207311877

Castells, M. (2014). El poder de las redes. En J. Godó (Ed.), Vanguardia Dossier (p. 6-13). Barcelona: La Vanguardia. Recuperado de https://www.researchgate.net/profile/Gustavo_Cardoso5/publication/301867379_Movilizacion_social_y_redes_sociales/links/572a5bo708ae2efbfdbc1f6c/Movilizacion-social-y-redes-sociales.pdf

Cavero, O. (julio de 2016). Después del Baguazo: informes, diálogo y debates. 13 (Serie Justicia y Conflictos n. 1). Recuperado de Cisepa: http://cisepa.pucp.edu.pe/wp-content/ uploads/2016/o7/baguazo_cavero.pdf

Christakis, N., \& Fowler, J. (2009). Connected: The Surprising Power of Our Social Networks and How They Shape Our Lives. Nueva York: Little, Brown and Co.

Collins, R. (1996). Cuatro tradiciones sociológicas. Iztapalapa: Universidad Autónoma Metropolitana.

Correa, G. (mayo de 2012). El concepto de mediación técnica en Bruno Latour. Psicología, Conocimiento y Sociedad, 2(1), 56-81. Recuperado de http://www.scielo.edu.uy/pdf/pcs/ v2n1/v2n1a04.pdf

Coser, L. (1961). Las funciones del conflicto social. Ciudad de México: Fondo de Cultura Económica.

Day, M. (2019). El concepto de red en Manuel Castells y Bruno Latour. El debate agencia-estructura en la teoría social sobre la red. RevIISE , 13, 69-76. Recuperado de https://www. researchgate.net/publication/332131119_El_concepto_de_red_en_Manuel_Castells_y_ Bruno_Latour_El_debate_agencia-estructura_en_la_teoria_social_sobre_la_red 
De Kerckhove, D. (s/f). Prólogo de Inteligencias en conexión. Hacia una sociedad de la web. Recuperado de Relato Digital Universidad Javeriana: https:/www.javeriana.edu.co/ relato_digital/r_digital/cibercultura/kerckhove1.htm

De la Cadena, M. (2015). Earth Beings: Ecologies of Practice Across Andean Worlds. New York: Duke University Press.

Defensoría del Pueblo. (enero de 2021). Reporte Mensual de Conflictos Sociales Nro. 203. Recuperado de Defensoría del Pueblo: https:/www.defensoria.gob.pe/wp-content/ uploads/2021/o2/Reporte-Mensual-de-Conflictos-Sociales-N\%C2\%Bo-2O3-enero-2021. pdf

Del Fresno, M. (2014). Haciendo visible lo invisible: visualización de la estructura de las relaciones en red en Twitter por medio del análisis de redes sociales. El profesional de la información, 23(3), 246-252. doi:http://dx.doi.org/10.3145/epi.2014.may.04

Dolorier, G., \& Paneque, P. (2013). Power, government, and territory: analysis of Bagua conflict, Peru. Letras verdes, (14), 139-159. doi:https://doi.org/10.17141/letrasverdes.14.2013.997

Domínguez, J., López, R., \& Ortega, J. (julio-diciembre de 2016). “Hagan un grupo de Facebook para esta clase". ¿De qué hablamos cuando hablamos de redes sociales? Revista Interamericana de Educación de Adultos, 116-126.

Espinoza, Ó. (2017). Los problemas indígenas amazónicos y sus demandas. En M. Ulfe, \& R. Trinidad (Eds.), En busca de reconocimiento (p. 119-136). Lima: Fondo editorial PUCP.

Fontanille, J. (2001). Semiótica del discurso. Lima: Fondo de Cultura Económica y Fondo Editorial de la Universidad de Lima.

Gascue, Á. (2009). El desafío de las nuevas tecnologías a los conceptos tradicionales de grupo primario y grupo de pertenencia. XXVII Congreso de la Asociación Latinoamericana de Sociología. VIII Latinoamericana de Sociología. 1-9. Recuperado de https://www. aacademica.org/o0o-062/95

González, N. (2014). La teoría de redes sociales y las políticas públicas. Forum(6), 81-97.

González-Palacio, L., González-Pala, M., \& Ortíz-Pabón, J. (octubre de 2017). Las redes sociales web como herramienta para gestionar información en procesos de co-creación. Risti, (24), 57-68. doi:10.17013/risti.24.57-68

Granovetter, M. (1973). The Strength of Weak Ties. American Journal of Sociology(6).

Guevara, V. (2011). Le conflit de Bagua: les problèmes du développement et les revendications des populations indigènes en Amazonie péruvienne. Droit et cultures: revue internationale interdisciplinaire, (62).

Guzmán, F. (2015). El problema de los medios: poder discursivo en el conflicto amazónico. VIII Seminario Regional (Cono Sur) ALAIC "Políticas, actores y prácticas de la comunicación: Encrucijadas de la investigación en América Latina (p. 147-180). Córdoba: Escuela de Ciencias de la Información (ECI) y Asociación Latinoamericana de los Investigadores de la Comunicación (ALAIC).

Guzmán, F. (2017). Medios de comunicación y representación de conflictos étnico-sociales en Perú: análisis crítico del discurso periodístico televisivo sobre el conflicto amazónico el "Baguazo". Tesis doctoral. Recuperado de https://www.tdx.cat/handle/10803/402572\#page $=1$

Guzmán, F., \& Rodrigo Alsina, M. (2019). El “Baguazo” en el discurso periodístico peruano. Un análisis crítico de los medios durante el conflicto amazónico de 2009. Estudios sobre el Mensaje Periodístico, 25(2), 853-867. doi:https://doi.org/10.5209/esmp.64812 
Hinojosa, D., Ricco, R., \& Toasa, A. (2013). La matanza de Bagua y los decretos ejecutivos. Letras verdes, (4), 21-23. doi:https://doi.org/10.17141/letrasverdes.4.2009.843

Latour, B. (2008). Reemsamblar lo social. Una introducción a la teoría del actor-red. Buenos Aires: Manantial.

Leff, E. (enero-marzo de 2011). Sustentabilidad y racionalidad ambiental: hacia "otro" programa de sociología ambiental. Revista Mexicana de Sociología 73(1), 5-46.

Lezama, J. (2004). La construcción del problema ambiental en la sociología. Ciudad de México: El Colegio de México AC. Centro de Estudios Demográficos y de Desarrollo Urbano. .

Li, F. (2015). Desenterrando el conficto. Empresas mineras, activistas y expertos en el Perú. Lima: IEP.

Llique, R., \& Santome, A. (2019). De "caníbales", "salvajes" y "terroristas antimineros" : la regulación de la libertad de expresión en el caso de los discursos de odio en los conflictos socioambientales. Tesis para optar la Licenciatura en Derecho. Lima: Pontificia Universidad Católica del Perú.

Manacés, J., \& Gómez, C. (2013). La verdad de Bagua. Informe en minoría de la Comisión Especial para investigar y analizar los sucesos de Bagua. Lima: Comisión de Derechos Humanos.

Marinas, L. (13 de abril de 2019). Instagram: Donde Millennials, Generación Z, Mcluhan y Bolter se cruzan. CIC. Cuadernos de Información y Comunicación, 24, 187-201. doi:https:// doi.org/10.5209/ciyc.64641

Martínez-Alier, J. (2015). Ecología política del extractivismo y justicia socio-ambiental. Interdisciplina, 3(7), 57-73.

Martins, P. (2009). Redes sociales: un nuevo paradigma en el horizonte sociológico. Cinta de Moebio, (35), 88-109. doi:10.4067/So717-554X2009000200002

Mendoza, M., \& Valenzuela, A. (2020). Las redes sociales en el Perú y la comunicación. En Y. Théorêt, \& P. Viallon (Eds.), La libertad de expresión en la era digital. Del "Fake News" a la inteligencia artificial (p. 448-472). Estrasburgo: Unesco.

Murray-Li, T. (2014). What is land? Assembling a resource for global investment. Transactions, 39(4), 589-602. doi:10.1111/tran.12065

Parra-Romero, A. (2020). Producción de conocimiento en conflictos socioambientales. Revista de Ciencias Sociales, 37-83. doi:10.36517/rcs.51.2.do2

Pérez Castro, J. (2005). Randall Collins y la tradición del conflictos: hacia la fundamentación de una teoría sociológica explicativa. Perspectivas docentes, 41-51. Recuperado de https:// dialnet.unirioja.es/servlet/articulo? codigo $=1704348$

Prestel, C. (2006). Reseñas. Reassembling the Social. Política y Sociedad, 43(3), 127-130.

Rheingold, H. (2004). Multitudes inteligentes. Barcelona: Gedisa.

Rodríguez, M. (2009). Las actuales protestas sociales y la demanda de ciudadanía y democracia participativa. PuntoEdu(150).

Romio, S. (2010). Bagua, un anno dopo. La figura del "politico indigeno" come nuova strategia mediatica del movimento indigeno peruviano. Confluenze, 2(2), 59-91. doi:https://doi. org/10.6o92/issn.2036-0967/1995

Sánchez-Duarte, J. M., \& Fernández-Romero, D. (2017). Subactivismo feminista y repertorios de acción colectiva digitales: prácticas ciberfeministas en Twitter. El profesional de la información, 26(5), 894-902. doi:http://doi.org/10.3145/epi.2017.sep.11

Simmel, G. (1986). Sociología. Estudios sobre las formas de socialización. Madrid: Alianza Editorial. 
Surrallés, A. (2011). Antropología despúes de Bagua. Movimiento indígena, políticas públicas $y$ conocimiento antropológico. Lima: Pontificia Universidad Católica del Perú.

Tarabini, S. (2019). Co-producción de conocimiento y redes en un conflicto socio-ambiental: una resistencia pionera. Revista de Paz y Conflictos, 12 (2), 177-197. doi:http://dx.doi. org/10.30827/revpaz.v12i2.10231

Tarrow, S. (1997). El poder en movimiento. Los movimientos sociales, la acción colectiva y la política. Madrid: Alianza Editorial.

Wellman, B. (1997). El análisis estructural de las redes sociales: del método y la metáfora a la teoría y la sustancia. Debate en Sociología(22), 47-97.

Zambrano, G. (2017). Bagua: entendiendo al derecho en un contexto culturalmente complejo. Lima: Pontificia Universidad Católica del Perú, Konrad-Adenauer-Stiftung \& Universidad Antonio Ruiz Montoya. 
